\title{
Importance des processus d'injection dans la formation de l'effet piézoélectrique du poly(fluorure de vinylidène)
}

\author{
M. Latour \\ Laboratoire de Physique Moléculaire et Cristalline, Centre de Dynamique des Phases Condensées (LA 233),
} Université des Sciences et Techniques du Languedoc, 34060 Montpellier Cedex.

(Reçu le 11 octobre 1979, révisé le 12 novembre, accepté le 26 novembre 1979)

\begin{abstract}
Résumé. - La technique des courants thermiquement stimulés associée à un procédé de découpage en surface des échantillons montre la dissymétrie de polarisation d'échantillons de $\mathrm{PVF}_{2}$. En particulier l'importance du processus d'injection des charges à partir de l'électrode positive lors de la formation de l'effet piézoélectrique est mise en évidence.
\end{abstract}

\begin{abstract}
Using TSC technique joined to a cutting process of the sample we show the asymmetric polarization of $\mathrm{PVF}_{2}$ samples and the importance of the injection process from the positive electrode, in the formation of the piezoelectric effect.
\end{abstract}

Introduction. - L'importance de l'interface Polymère-métal, dans la formation de l'effet piézoélectrique du polyfluorure de vinylidène, a été mise en évidence dans divers travaux, particulièrement par ceux de Sussner et Dransfeld [1], en utilisant des techniques de détection ultra sonores et optiques, ainsi que par ceux, antérieurs, de Murayama [2]. Nous avons ici utilisé la technique des courants thermiquement stimulés, associés à un procédé de découpage en surface des échantillons. Ces résultats confirment l'importance de la nature des contacts polymère-métal au cours de la polarisation du poly(fluorure de vinylidène) à bas champ, puisque nous avons ici employé des champs de l'ordre de $\pm 10 \mathrm{kV} / \mathrm{cm}$. Il est intéressant de noter qu'à champs plus intenses, la dissymétrie de la polarisation interne $\mathrm{du}$ diélectrique subsiste. Nous avons montré précédemment [3], en établissant la topographie des potentiels internes du $\mathrm{PVF}_{2}$ par des méthodes spectroscopiques, une différence très nette de la polarisation entre l'électrode positive et négative. Ces différences apparaissent également par corona, le corona positif donnant une polarisation différente du corona négatif.

Le poly(fluorure de vinylidène) présente des propriétés piézoélectriques et pyroélectriques lorsqu'il est polarisé par application d'une tension continue soit par corona soit entre deux électrodes.

Deux types de polarisations peuvent être induites, l'une liée à l'effet dipolaire, disparaît presque totalement après le premier cycle thermique, l'autre est thermo-réversible [4], si l'échantillon n'est pas chauffé excessivement.

Nous présentons les résultats obtenus par enregistrement des courants thermiquement stimulés de thermoélectrets de polyfluorure de vinylidène découpés après leur polarisation.

Ce procédé de découpage joint à la technique des C.T.S., apporte des précisions sur le processus de polarisation du poly(fluorure de vinylidène) et la contribution d'effets de charge d'espace liés à l'injection.

Procédé expérimental. - Les films de $55 \mu$ d'épaisseur ont été fournis par Kureha Chem. So, ils présentent la configuration de la phase cristalline $\beta$ leur densité : $1,78 \mathrm{~g} / \mathrm{cm}^{3}$, cristallinité : $38 \%$ mesurée par rayons $X$. En infrarouge le rapport d'absorption des bandes $510 \mathrm{~cm}^{-1}$ et $530 \mathrm{~cm}^{-1}$ est 0,02 . La masse moléculaire est voisine de 450000 .

Divers types de contacts aux électrodes ont été utilisés que nous appellerons injectants lorsqu'un dépôt métallique argent ou carbone est réalisé sur le polymère, et bloquant lorsqu'on interpose un film de téflon de $6 \mu$ entre le polymère et l'électrode métallique (argent ou carbone). Les dimensions de l'échantillon sont : $0,8 \times 0,5 \mathrm{~cm}$. La température de polarisation est $110^{\circ} \mathrm{C}$, l'intensité du champ polarisant est faible : $\pm 10 \mathrm{kV} / \mathrm{cm}$ le temps de polarisation : $10 \mathrm{~min}$ à $80^{\circ} \mathrm{C}$ et pendant les refroidissements jusqu'à $-100^{\circ} \mathrm{C}$, à la vitesse de $10^{\circ} \mathrm{C} / \mathrm{min}$. 
Le champ polarisant est supprimé à $-100^{\circ} \mathrm{C}$, l'échantillon est ensuite refroidi à la température de l'azote liquide puis découpé au microtome à cette température (Fig. 1). Pour chaque système d'électrodes nous avons considéré trois échantillons identiques, polarisés dans les mêmes conditions, pour deux d'entre eux l'épaisseur a été réduite respectivement de 5 et $10 \mu$. Les C.T.S. sont enregistrés sur les 3 échantillons en les réchauffant à la vitesse de $4^{\circ} \mathrm{C} / \mathrm{min}$ sans potentiel appliqué, entre des électrodes métalliques carbone ou argent. Les courants sont détectés par un picoampèremètre type Keithley. L'intensité des courants enregistrés est de l'ordre de $10^{-11} \mathrm{~A}$ à basse température, et de $10^{-9} \mathrm{~A}$ par effet piézoélectrique lorsque la température dépasse $60^{\circ} \mathrm{C}$. Le coefficient piézoélectrique statique transverse est mesuré à température ambiante par pression normalement au plan du film. Les potentiels de surface sont mesurés, également à température ambiante, à l'aide du fieldmetre type Monroe Electronic. Ces mesures sont effectuées environ $30 \mathrm{~min}$ après l'arrêt de la tension de polarisation.

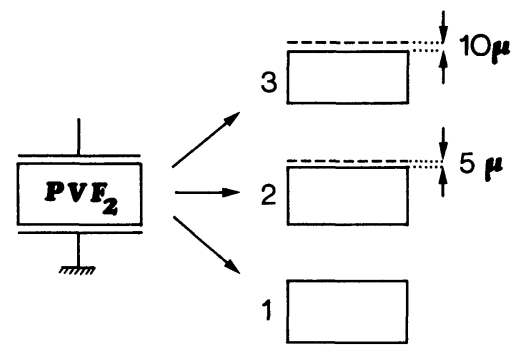

Fig. 1. - Procédé de polarisation et de découpage des échantillons.

[Poling and cutting process for PVF $_{2}$ samples.]

Résultats. - La technique conventionnelle des C.T.S. n'est pas suffisante pour analyser le type de polarisation du polyfluorúre de vinylidène. La figure 2 donne l'allure générale des C.T.S. enregistrés sur le $\mathrm{PVF}_{2}$ polarisé. La mesure est faite entre $-100^{\circ} \mathrm{C}$ et $+160^{\circ} \mathrm{C}$. L'échantillon étant polarisé entre deux électrodes métalliques (Ag). Nous observons 3 pics : à basse température : à $-51^{\circ} \mathrm{C}$ un pic bien défini dans la région de la transition vitreuse, et deux pics ou épaulements à $+60^{\circ} \mathrm{C}$ et $+110^{\circ} \mathrm{C}$. Dans cette région, l'effet pyroélectrique ne permet pas une bonne définition des pics. Après un premier cycle thermique, nous observons (ligne pointillée Fig. 2) que les trois pics semblent disparaître presque complètement. A haute température la ligne de base des diagrammes ne reste pas constante. Le maximum défini à $-51^{\circ} \mathrm{C}$ a été bien analysé par les travaux de Pfister Abkowitz et al. $[5,6]$. Ce pic serait dû à la réorientation des dipôles, avec une énergie'd'activation apparente de $3,6 \pm 0,4 \times 10^{4} \mathrm{~K}$ et une fréquence de relaxation

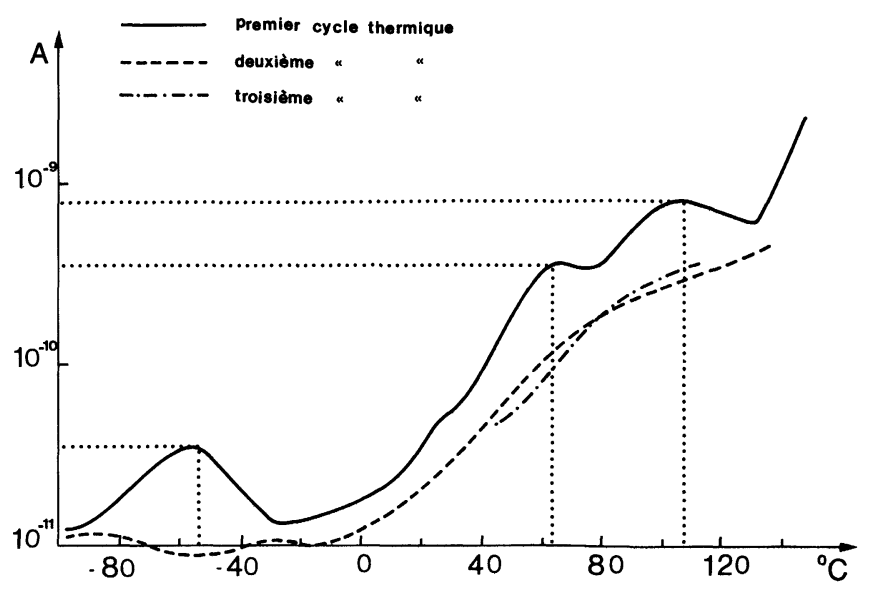

Fig. 2. - Forme classique des courbes de C.T.S. d'un échantillon de $\mathrm{PVF}_{2}$ polarisé dans les conditions de l'expérience. Les trois courbes successives correspondant à trois cycles thermiques successifs.

[TSC classical curves of poled $\mathrm{PVF}_{2}$ between $-100^{\circ} \mathrm{C}$ and $+160^{\circ} \mathrm{C}$ : continue line : first heating cycle ; dashed lines : second and third heating cycles.]

de $2 \times 10^{-3} \mathrm{~s}^{-1}$ pour le processus de réalignement dominant. Pour les deux épaulements à $60^{\circ} \mathrm{C}$ et $110{ }^{\circ} \mathrm{C}$ l'analyse classique des C.T.S. paraît difficile; la ligne de base étant fluctuante du fait de la présence de l'effet pyroélectrique. Le processus de découpage apporte une simplification à ce problème, en localisant spacialement la contribution des charges.

A. Cas des électrodes « injectantes ». - 1. TENSION DE POLARISATION POSITIVE. - Les trois échantillons sont polarisés par une tension positive sur la face supérieure (celle qui, pour deux d'entre eux, sera découpée). Les échantillons sont ensuite découpés comme indiqué précédemment. Les courbes de C.T.S. obtenues pour les trois échantillons sont représentées sur la figure 3. Le maximum des charges d'espace

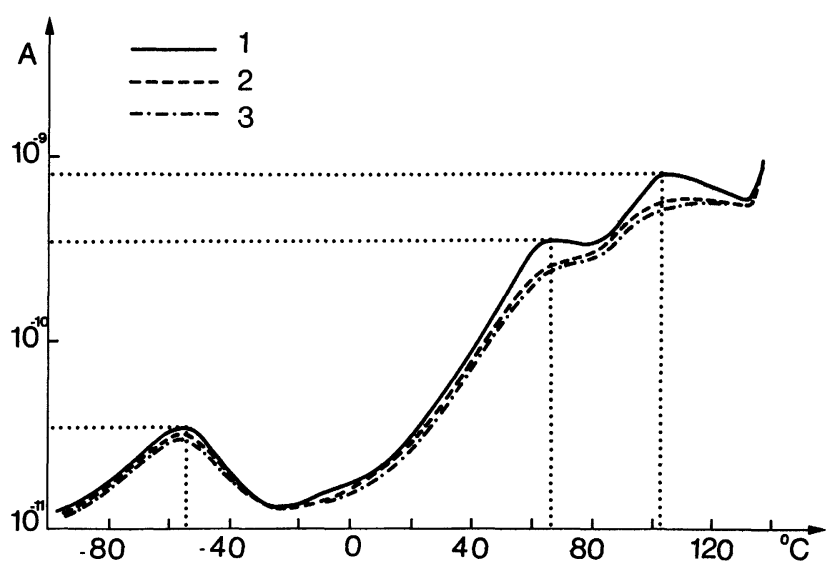

Fig. 3. - C.T.S. des trois échantillons après polarisation par une tension positive et contacts « injectants ".

[TSC curves of $\mathrm{PVF}_{2}$ samples poled with positive field and injecting contact.] 
semble localisé près de l'électrode positive, la suppression de cette région modifiant en effet fortement le profil des courbes particulièrement dans la région des deux épaulements. Le pic à $-51^{\circ} \mathrm{C}$ n'est pas modifié, nous supposons que la polarisation par orientation dipolaire est pratiquement homogène dans tout le volume du diélectrique alors qu'une polarisation type charge d'espace serait responsable en partie de la présence des pics à $60^{\circ}$ et $110^{\circ} \mathrm{C}$.

A température ambiante nous observons une homocharge apparente sur la face supérieure de l'échantillon $(V=+270 \mathrm{~V})$ avec un coefficient piézoélectrique de $2 \mathrm{pc} / \mathrm{N}$; après quelques heures, une hétérocharge stable apparaît avec un potentiel de surface $V=-80 \mathrm{~V}$ et une valeur du coefficient piézoélectrique statique transverse égale à $1,8 \mathrm{pc} / \mathrm{N}$, inchangé après 6 mois.

2. Tension DE POlaRisation NÉGATIVE. - Les trois échantillons sont polarisés par une tension négative appliquée sur l'électrode supérieure puis découpés. Les courbes de C.T.S. obtenues sur les trois échantillons sont représentées sur la figure 4. On observe que dans ce cas, contrairement au cas précédent, aucun changement important n'apparaît dans la configuration des courbes. Nous pouvons en

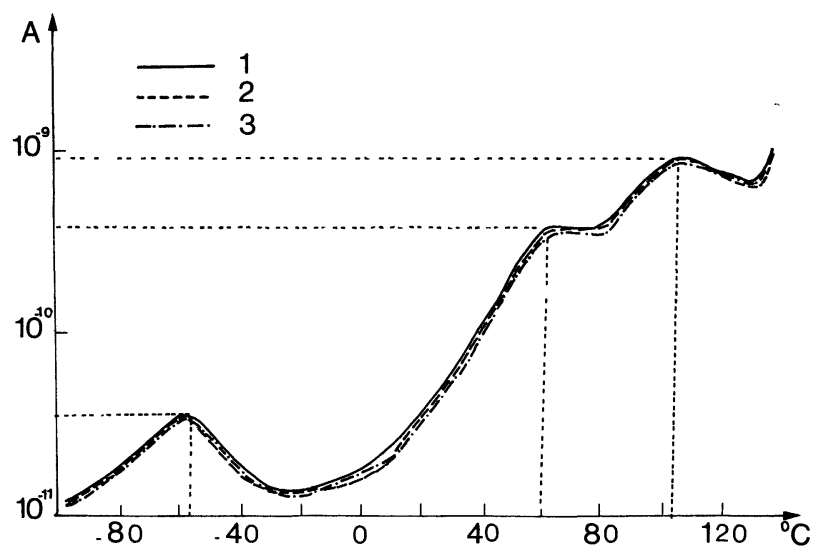

Fig. 4. - C.T.S. des trois échantillons après polarisation par une tension négative et contacts « injectants».

[TSC curves of $\mathrm{PVF}_{2}$ samples poled with negative field and injecting contact.]

déduire qu'il n'y a pas d'effet important de charge d'espace au voisinage de l'électrode supérieure puisque la suppression de cette partie du diélectrique ne modifie pas le profil des courbes. A température ambiante l'homocharge initiale apparente est $V=-280 \mathrm{~V}$, évolue vers une hétérocharge stable de $+70 \mathrm{~V}$; le coefficient piézoélectrique statique transverse $=2,2 \mathrm{pc} / \mathrm{N}$ évolue et tend vers une valeur stable de $1,9 \mathrm{pc} / \mathrm{N}$.
B. Cas des électrodes « bloquantes ». - 1. TENSION DE POLARISATION POSITIVE. - Les trois échantillons sont polarisés et découpés comme précédemment avec une tension positive sur l'électrode supérieure, un film de Téflon PTFE étant intercalé entre l'électrode et le diélectrique. Le potentiel est corrigé pour garder la même valeur appliquée au diélectrique. Les courbes de C.T.S. obtenues pour les trois échantillons, après découpage, sont indiquées sur la figure 5 .

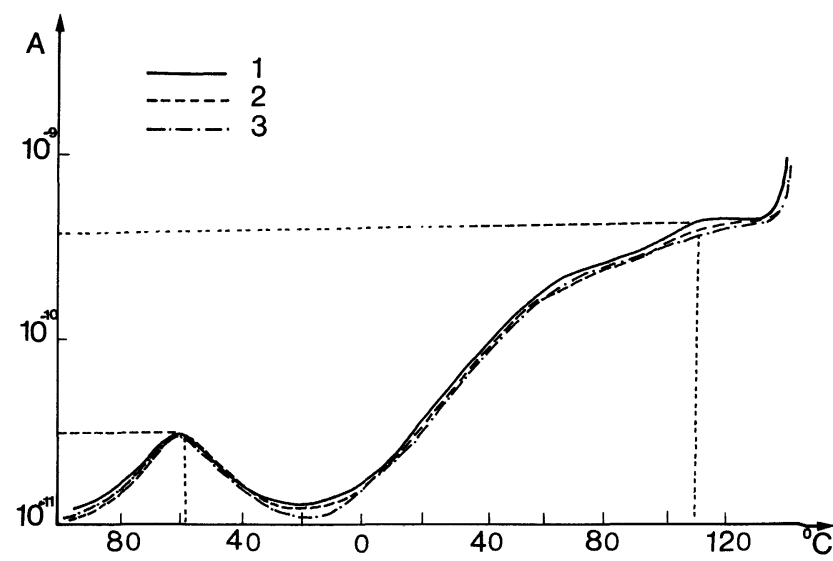

Fig. 5. - C.T.S. des trois échantillons après polarisation par une tension positive et contacts « bloquants ».

[TSC curves of $\mathrm{PVF}_{2}$ samples poled with positive field and blocking contact.]

Nous constatons que la position en température des différents maxima est très légèrement décalée vers les basses températures. Ce décalage serait attribué à la répartition non uniforme de la température dans le $\mathrm{PVF}_{2}$ lié à la présence du film de Téflon. On constate également que l'amplitude du pic à $-51^{\circ} \mathrm{C}$ est très légèrement inférieure mais pratiquement semblable à celle obtenue sur les courbes précédentes, par contre les maxima à $60^{\circ} \mathrm{C}$ et $110^{\circ} \mathrm{C}$ sont pratiquement inexistants. Ceci confirmerait l'hypothèse d'une orientation dipolaire, relaxant à basse température, pratiquement homogène dans tout le volume du diélectrique, et un effet de charge d'espace relaxant à $60^{\circ} \mathrm{C}$ et $110^{\circ} \mathrm{C}$, associés à un effet d'injection par l'électrode positive.

A température ambiante l'hétérocharge initiale égale à $V=-400 \mathrm{~V}$ et le coefficient piézoélectrique statique transverse de $0,6 \mathrm{pc} / \mathrm{N}$ décroissent tous deux fortement vers des váleurs stables, $V=-15 \mathrm{~V}$ et $0,2 \mathrm{pc} / \mathrm{N}$ extrêmement faibles. Nous devons remarquer la relation entre la valeur du coefficient piézoélectrique et l'injection des charges à partir de l'électrode positive. Lorsque cette injection n'existe pas, la valeur du coefficient piézoélectrique reste très faible, il est par ailleurs toujours associé à une hétérocharge. 
2. Tension de polarisation NÉGative. - Les échantillons sont polarisés par une tension négative appliquée sur l'électrode supérieure avec interposition du film de téflon du côté de cette électrode seulement. Les courbes de C.T.S. obtenues pour les trois échantillons après découpage sont indiquées sur la figure 6 . Les remarques relatives à la position des pics, faites dans le cas précédent, restent valables ici. Les deux

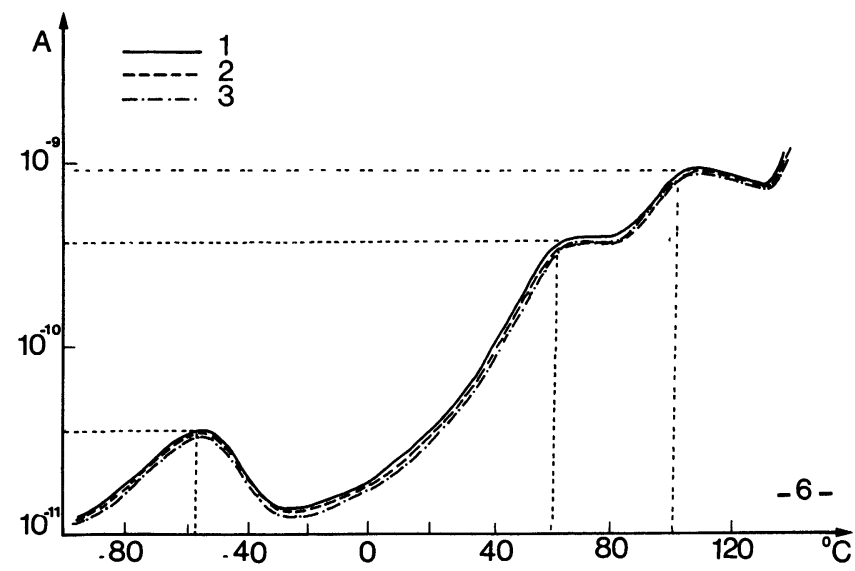

Fig. 6. - C.T.S. des trois échantillons après polarisation par une tension négative et contacts «bloquants».

[TSC curves for $\mathrm{PVF}_{2}$ samples poled with negative bias field and blocking contact.] épaulements à $60^{\circ} \mathrm{C}$ et $110^{\circ} \mathrm{C}$ sont très nets. $\mathrm{Si}$ nous supposons que ces pics sont liés à la présence d'une charge d'espace près de l'électrode positive, les résultats du diagramme dans ce cas confirmeraient encore cette hypothèse.

Conclusion. - Par la technique des courants thermiquement stimulés, associée à un processus de découpage, nous avons montré expérimentalement la contribution de l'orientation dipolaire et du processus d'injection de charges dans le polyfluorure de vinylidène, de forme cristalline $\beta$ polarisé.

- A basse température les résultats confirment les travaux effectués par Pfister et Abkowitz, le pic à - $51^{\circ} \mathrm{C}$ semble associé, pour sa part la plus importante, aux fluctuations des dipôles permanents et pour une très faible part à un effet de charge d'espace.

- A haute température, deux pics apparaissent à $60^{\circ} \mathrm{C}$ et $110^{\circ} \mathrm{C}$. Leur présence et la valeur du coefficient piézoélectrique statique transverse semblent fortement liées à la nature du contact aux électrodes.

Nous montrons en particulier l'importance du processus d'injection des charges à partir de l'électrode positive dans la formation de ces pics et dans l'amplitude de l'effet piézoélectrique. Tous ces résultats sont résumés tableau I. Ils sembleraient confirmer, par une méthode différente, les résultats obtenus par Sussner et Dransfeld.

Tableau I. - Résumé des résultats montrant la valeur du coefficient piézoélectrique statique transverse et la nature de la charge en fonction des conditions de la polarisation.

[Summary of the results showing the value of piezoelectric coefficient and charge nature, function of poling conditions.]

\section{Conditions de polarisation nature du contact}

Nature et valeur des potentiels de surface

Valeur du coefficient piézoélectrique statique transverse

Injectant
coté positif
-
homocharge
$+270 \mathrm{~V}$
évolue vers
$-80 \mathrm{~V}$

$2,2 \mathrm{pc} / \mathrm{N}$ évolue vers $1,9 \mathrm{pc} / \mathrm{N}$

$$
\begin{aligned}
& \text { Injectant } \\
& \text { coté négatif } \\
& - \\
& \text { homocharge } \\
& -280 \mathrm{~V} \\
& \text { évolue vers } \\
& +70 \mathrm{~V}
\end{aligned}
$$

$2,2 \mathrm{pc} / \mathrm{N}$ évolue vers $1,9 \mathrm{pc} / \mathrm{N}$

$$
\begin{aligned}
& \text { Bloquant } \\
& \text { coté positif } \\
& - \\
& \text { hétérocharge } \\
& -400 \mathrm{~V} \\
& \text { décroît vers } \\
& -15 \mathrm{~V}
\end{aligned}
$$

$0,8 \mathrm{pc} / \mathrm{N}$ décroît $0,2 \mathrm{pc} / \mathrm{N}$
Bloquant coté négatif homocharge $-100 \mathrm{~V}$ évolue vers $+50 \mathrm{~V}$

$2,1 \mathrm{pc} / \mathrm{N}$ évolue vers $1,8 \mathrm{pc} / \mathrm{N}$

\section{Bibliographie}

[1] Sussner, H. et Dransfeld, K., J. Polym. Sci. Polym. Phys. Ed. 16 (1978) 529-543.

[2] Murayama, N., J. Polym. Sci. Polym. Phys. Ed. 13 (1975) 929.

[3] LatouR, M., J. of Electrost. No 2 (1977) 241-248. Traduction en anglais parue dans le journal du British Post Office Research Centre. Marthesham Heath Ipswich (G.B.) No 3471 .

[4] Burckard, H. et Pfister, M., J. Appl. Phys. 45 (1974) 3360.
[5] Pfister, G., Abkowitz, M., Crystal, R. G., J. Appl. Phys. 44 (1973) 2064.

[6] Abkowitz, M., Luca, P. J., Pfister, G., Prest, W. M., Communication dans le proceeding of Piezoelectric and Pyroelectric Symposium Workshop, N.B. April 1975 Gaithersburg (U.S.).

[7] Pfister, G., Abkowitz, H., Crystal, R. G., J. Appl. Phys. 44 (1973) 2064. 